\title{
Modélisation mathématique du séchage dans un four (air chaud) de tranches de mangue (Mangiféra indica $\mathrm{L}$.)
}

\author{
N'guessan Verdier ABOUO ${ }^{1,3^{*}}$, Alhassane FOFANA ${ }^{2}$, Yevi Delphine N'GUESSAN ${ }^{1}$ et \\ Nogbou Emmanuel ASSIDJO ${ }^{3}$
}

\author{
${ }^{1}$ Laboratoire de Biotechnologie, Agriculture et Valorisation des Ressources Biologiques (LBAVRB), UFHB, UFR \\ Biosciences, 22 BP 582 Abidjan 22, Côte d'Ivoire. \\ ${ }^{2}$ Laboratoire de Physique de l'Atmosphère et de Mécanique des Fluides (LAPAMF), UFHB, UFR Sciences et Structure de la \\ Matière et de la Technologie (SSMT), 22 BP 582 Abidjan 22, Côte d'Ivoire. \\ ${ }^{3}$ Laboratoire des Procédés Industriels de Synthèse de l'Environnement et des Energies Nouvelles (LAPISEN), INPHB, DFR \\ GCAA, BP 1093 Yamoussoukro, Côte d'Ivoire. \\ *Auteur correspondant, E-mail: v.abouo@gmail.com; Tél : (+225) 01409000
}

\section{RESUME}

Ce travail vise à étudier le séchage de la mangue à différentes épaisseurs et températures en vue de valorisation. Les expériences ont été menées à partir d'une matrice factorielle par la méthode gravimétrique statique. Les valeurs expérimentales ont été ajustées à huit modèles empiriques par la méthode du gradient afin d'estimer les paramètres et coefficients des modèles. Les critères statistiques que sont le $\mathrm{R}^{2}$, l'erreur quadratique moyenne $(\mathrm{EQM})$ et le $\chi^{2}$ ont servi à justifier le choix du modèle de séchage. Le séchage a duré 16-18;23-27 et 29-33 minutes respectivement aux températures 60,50 et $40^{\circ} \mathrm{C}$ pour les épaisseurs 1,5 et $1 \mathrm{~cm}$. Le modèle logarithmique est retenu et présente les valeurs du $\mathrm{R}^{2}(0,9984 ; 0,9984 ; 0,9981 ; 0,9987 ; 0,9988 ; 0,9985)$ les plus élevées et les plus faibles valeurs de $\chi^{2}(2,43$ e- $07 ; 1,58$ e-07 ; 1,25 e-06 ; 2,02 e-07 ; 4,56 e-06 et 1,37 e06) et EQM $(2,42$ e-04; 1,95 e-07 ; 5,47 e-04 ; 2,20 e-04; 0,0010 et 5,69 e-04). Le coefficient de diffusion varie de 8,615 e-09 à 9,155 e- $09 \mathrm{~m}^{2} / \mathrm{s}$ et dépend fortement de la température de séchage. L'énergie d'activation est estimée entre 22,430 - 25,358 KJ / mol.

(C) 2020 International Formulae Group. All rights reserved.

Mots clés : Séchage, mangue, modèle, coefficient de diffusion, énergie d'activation.

\section{Mathematical modeling of the drying in an oven (hot air) of slices of mango (Mangifera indica L.)}

\begin{abstract}
This work aims to study the drying of mango at different thicknesses and temperatures a view to valorization. The experiments were carried out from a factor matrix by the static gravimetric method. The experimental values were fitted to eight empirical models by the gradient method in order to estimate the parameters and coefficients of the models. The statistical criteria of $\mathrm{R}^{2}$, mean square error (MSE) and $\chi^{2}$ were used to justify the choice of the drying model. Drying lasted 16-18; 23-27 and 29-33 minutes respectively at temperatures 60,50 and $40{ }^{\circ} \mathrm{C}$ for thicknesses 1.5 and $1 \mathrm{~cm}$. The logarithmic model is retained and presents the highest values of $\mathrm{R}^{2}(0.9984 ; 0.9984 ; 0.9981 ; 0.9987$; $0.9988 ; 0.9985)$ and the lowest values of $\chi^{2}(2.43$ e- 07 ;
\end{abstract}


1.58 e-07; 1.25 e-06; 2.02 e-07; 4.56 e-06 and 1.37 e-06) and MSE (2.42 e-04; 1.95 e-07; 5.47 e-04; 2.20 e-04; 0.0010 and $5.69 \mathrm{e}-04)$. The diffusion coefficient varies from $8.615 \mathrm{e}-09$ to $9.155 \mathrm{e}-09 \mathrm{~m}^{2} / \mathrm{s}$ and strongly depends on the drying temperature. The activation energy is estimated to be $22.430-25.358 \mathrm{KJ} / \mathrm{mol}$.

(C) 2020 International Formulae Group. All rights reserved.

Keywords : Drying, mango, models, diffusion coefficient, activation energy.

\section{INTRODUCTION}

L'économie de la Côte d'Ivoire repose sur l'agriculture, qui est favorisée par un climat chaud et humide (Kakou et al., 2015). Elle est dominée par l'exportation de produits dit de rente, en particulier le cacao, le café, le palmier à huile, l'hévéa etc... (Akmel et al., 2008). La Côte d'Ivoire exporte également des fruits et légumes aux nombres desquels les plus importants (quantitativement) sont respectivement l'ananas, la banane et la mangue (CBI-MFA, 2019).

Ces trois produits contribuent pour près de $4 \%$ à la formation du produit intérieur brut (PIB) (Nouar et al., 2013). Avec une production avoisinant 160000 tonnes dont plus de 33000 tonnes exportées en Europe, la production ivoirienne s'est hissée au troisième rang des fournisseurs de mangues du marché européen après le Brésil et le Pérou (CBIMFA, 2019). La vente de la mangue rapporte au pays plus de 6,5 à 7 milliards de FCFA aux producteurs (Assovie, 2012). Le niveau d'exportation de la mangue est estimé à environ $30 \%$ de sa production et près de $30-60 \%$ de pertes post-récoltes enregistrées du fait des difficultés d'écoulement liées à l'étroitesse du marché intérieur, la courte durée de conservation de la mangue et les maladies des fruits (CBI-MFA, 2019).

La presque quasi-totalité des pertes post-récoltes de produits agricoles trouvent une solution par la transformation en d'autres sousproduits ou par la réduction de la biodisponibilité en eau notamment le séchage. C'est une technique ancienne de conservation des produits agricoles (Akmel et al., 2008). Il consiste à réduire, suivre et contrôler la disponibilité en eau dans un produit (Nogbou et al., 2015). Cette biodisponibilité en eau dans le produit entraine des réactions biochimiques complémentaires (Afoakwa et al., 2008). A cet effet, le séchage constitue une étape cruciale du traitement post-récolte (Afoakwa et al., 2013 ; Kouadio et al., 2015). Il s'effectue généralement au soleil (séchage naturel), moins onéreux et par des techniques de séchage artificiel (Abouo et al., 2015a). Comme technique de séchage artificiel, il y a la conduction qui s'effectue généralement dans des fours (conduction et rayonnement) à des températures et/ou puissances spécifiques (Koné, 2011 ; Abouo et al., 2015b). Lors du séchage, la perte en eau du produit peut s'effectuer par pesée gravimétrique statique (Akmel et al., 2008). Cette technique permet de déterminer la durée du séchage, la quantité totale d'eau libre à enlever du produit afin de la rendre très peu ou pas disponible dans le produit (Akmel et al., 2009). De ce fait, elle permet d'établir les courbes caractéristiques de séchage (CCS) qui expriment l'évolution de la vitesse de séchage en fonction de la teneur réduite en eau du produit et d'en déduire les modèles mathématiques susceptibles d'être lissés aux courbes de séchage dudit produit (Hii et al., 2006). Les modèles mathématiques définis empiriquement dans la littérature sont nombreux et permettent de simuler expérimentalement le séchage (Hii et al., 2006). A partir des informations sur le transfert de l'humidité pendant le séchage qui est contrôlé par la diffusion interne (deuxième loi de Fick), le coefficient de diffusion et l'énergie d'activation peuvent en être déduits pour une connaissance parfaite d'importantes propriétés physiques et les conditions d'entreposage du produit en vue de conservation (Touati, 2008 ; Bonazzi et al., 2008 ; Koné, 2011, Krysiak et al., 2011).

Il existe des travaux de séchage de la mangue en Côte d'Ivoire, au Burkina Faso et au Ghana. Aussi, des travaux de recherche ont été réalisés sur les principaux microorganismes responsables de l'anthracnose, maladie dévastatrice du manguier (Yah, 2015; 
Dembele et al., 2019) sans oublier ceux relatifs aux procédés de déshydratation osmotique (Floury et al., 2008; N'Goran et al., 2012). Concernant le séchage de la mangue, la littérature fait mention de l'usage de séchoir type solaire par convection forcée (Mamouda et al., 2019). Face aux nombreuses pertes postrécoltes enregistrées de la production, la très faible quantité de données scientifiques disponibles à ce sujet et les progrès scientifiques réalisés à partir du séchage de diverses matrices agricoles, il parait opportun de mener des investigations afin de contribuer à apporter des solutions à la problématique des pertes post-récoltes de la mangue. Ces investigations consisteront à caractériser le séchage de la mangue par la modélisation (empirique), à quantifier les paramètres tels que l'énergie d'activation et le coefficient de diffusion de l'eau au cours du séchage des tranches de mangue.

\section{MATERIEL ET METHODES \\ Préparation de l'échantillon}

Le matériel biologique est constitué de dix (10) kilogrammes de mangue de variété 'kent", acheté au grand marché de la commune d'Abobo (Abidjan nord) dans la période d'Avril 2020 et transporté au laboratoire pour la suite des travaux. Les mangues ont été épluchées, tranchées en épaisseur de 1 et $1,5 \mathrm{~cm}$. Des échantillons de tranches de mangue de $5 \mathrm{~g}$ ont été constitués.

\section{Procédure de séchage de la mangue}

Les tranches obtenues ont été mises à sécher dans un four à moufle (Thermoscientific) à trois (03) différentes températures selon un plan d'expérience de degré un comme indiqué dans le Tableau 1 (domaine expérimental) (Chekroune, 2009). Les essais de séchage ont été menés et la perte différentielle de masse de l'échantillon a été effectuée par pesée gravimétrique statique à l'aide d'une balance (Sartorius, A200S, France) jusqu'à ce que la différence entre trois (03) pesées successives (chaque heure) n'excède la valeur de 0,001 (Belhamidi et al., 1993). En fin de séchage, la teneur en eau résiduelle a été déterminée selon la méthode AOAC (2005).

\section{Etablissement de courbes caractéristiques} de séchage (CCS)

Les CCS ont été établies en représentant les vitesses de séchage normées en fonction des teneurs en eau réduites. Elles permettent d'obtenir les équations de vitesses de séchage sous la forme de polynômes de degré 3 (Abouo et al., 2015b). Les expressions mathématiques des différentes vitesses de séchage sont les suivantes :

$$
f=\left[\frac{\left(\frac{-d X}{d t}\right)}{\left(\frac{-d X}{d t}\right)_{n}}\right]=A X^{* 3}+\mathbf{B} X^{* 2}+\mathbf{C} X^{*}+D
$$

Avec : A, B, C, D des coefficients à déterminer

\section{Modélisation mathématiques du séchage des tranches de mangue}

Les teneurs en eau d'équilibre des échantillons séchés (tranches sèches de mangue) ont été déterminées à partir des équations suivantes :

$$
\begin{aligned}
& X^{*}=\frac{X_{t}-X_{e}}{X_{0}-X_{e}} \\
& \frac{d X}{d t}=\frac{X_{t+d t}-X_{t}}{d t}
\end{aligned}
$$

Avec: $X_{t}$ : teneur en eau en instant $t ; X_{e}$ : teneur en eau d'équilibre ; $X_{0}$ : teneur en eau initiale (Tableau 2).

Le Tableau 3 présente des modèles empiriques décrits dans la littérature utilisées pour simuler la cinétique de séchage des produits végétaux. Au nombre de huit, retenus pour ce travail, ces modèles contiennent des paramètres et coefficients à déterminer à partir des données expérimentales du séchage (Akmel et al., 2009). La méthode du gradient a été développée sous un programme de calcul puis implémenté pour estimer les coefficients et paramètres à l'aide du logiciel MatLab R2016a (Abouo et al., 2015b). Les critères statistiques d'appréciation du lissage des données expérimentales aux différents modèles empiriques décrits dans la littérature ont été retenus pour le coefficient de détermination multiple $\mathrm{R}^{2}$ proche de l'unité et pour de faibles valeurs de l'erreur quadratique moyen (EQM) et $\chi^{2}$ proches de zéro (Nogbou et al., 2015 ; Karidioula et al., 2018). 
Tableau 3 : Modèles de séchage utilisés.

\section{MODELES EQUATIONS MATHEMATIQUES REFERENCES}

\begin{tabular}{rcr}
\hline $\begin{array}{r}\text { Approche- } \\
\text { Diffusion }\end{array}$ & $X^{*}=a \exp ^{(-k t)}+(1-a) \exp ^{(-k b t)}$ & Gowen et al., 2008 \\
Deux-Termes & $X^{*}=a \exp ^{\left(-k_{0} t\right)}+b \exp ^{\left(-k_{1} t\right)}$ & Roberts et al., 2008 \\
\hline $\begin{array}{r}\text { Henderson et } \\
\text { Pabis modifié }\end{array}$ & $X^{*}=a \exp ^{(-k t)}+b \exp ^{(-g t)}+c \exp ^{(-h t)}$ & Abouo et al., 2015b \\
\hline Logarithmique & $X^{*}=a \exp ^{(-k t)}+\mathrm{c}$ & Togrul et Pehlivan, 2004 \\
\hline Middili et Kucuk & $X^{*}=a \exp ^{\left(-k t^{n}\right)}+b t$ & Midilli et Kucuk, 2003 \\
\hline Newton & $X^{*}=\exp ^{(-k t)}$ & Yaldiz et al., 2001 \\
\hline Page & $X^{*}=\exp ^{\left(-k t^{n}\right)}$ & Jurendic, 2012 \\
\hline Verma et al., & $X^{*}=a \exp ^{(-k t)}+(1-a) \exp ^{(-g t)}$ & Verma et al., 1985 \\
\hline
\end{tabular}

Avec : a, b, c, g, h, k, k0, k 1 paramètres et coefficients à déterminer.

Les critères statistiques de choix des modèles ont été :

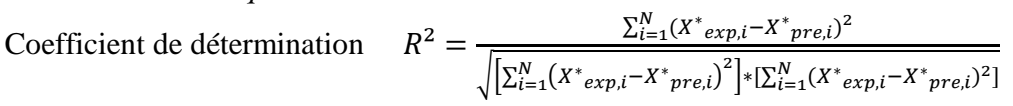

Khi carré réduit : $\quad \quad \chi^{2}=\frac{\sum_{i=1}^{N}\left(X^{*} \text { exp, },-X^{*}{ }_{p r e, i}\right)^{2}}{N-z}$

Erreur Quadratique Moyenne : $E Q M=\left[\frac{1}{2} \sum_{i=1}^{N}\left(X^{*}{ }_{\text {exp }, i}-X^{*}{ }_{\text {pre }, i}\right)^{2}\right]^{\frac{1}{2}}$

(Sorour et El-Mesery, 2014 ; Kakou et al., 2015)

\section{Estimation de la diffusivité massique et de l'énergie d'activation}

Le transfert de l'humidité pendant le séchage est contrôlé par la diffusion interne. La deuxième loi de Fick de diffusion a été largement utilisée pour décrire le processus de séchage pour la plupart des produits biologiques liant la teneur en eau du produit (M), la diffusivité massique (D), le temps (t) et la direction (z) (Touati, 2008 ; Akmel et al., 2009).

$$
\begin{aligned}
& M R=\frac{M}{M_{0}}= \\
& \frac{8}{\pi^{2}} \sum_{i=1}^{\infty} \frac{1}{(2 n-1)} \operatorname{Exp}\left(-\frac{\left.(2 n-1)^{2} \pi^{2} D_{e f f} t\right)}{4 L^{2}}\right)
\end{aligned}
$$

Avec : $M R$ est la teneur en eau réduite, $D_{\text {eff }}$ est le coefficient de diffusivité effectif $(\mathrm{m} / \mathrm{s})$; L est la demi-épaisseur de la tranche $(\mathrm{m})$ et $\mathrm{n}$, un entier positif.

Pour une longue période de séchage, l'équation ci-dessus peut être simplifiée, en prenant le logarithme naturel des deux côtés. La précédente équation devient :

$\operatorname{Ln} M R=\operatorname{Ln} \frac{8}{\pi^{2}}-\left(\frac{\pi^{2} D_{e f f} t}{4 L^{2}}\right)$

Le coefficient de diffusion dépend de la température et est décrit par la loi d'Arrhenius selon l'équation suivante :

$D_{\text {eff }}=D_{0} \operatorname{Exp}\left(-\frac{E_{a}}{R T}\right)$ 
$D_{0}$ est la constante de l'équation d'Arrhenius $\left(\mathrm{m}^{2} / \mathrm{s}\right)$; Ea est l'énergie d'activation $(\mathrm{kJ} / \mathrm{mol}) ; \mathrm{T}$ est la température de l'air de séchage (K) et $\mathrm{R}$ est la constante de gaz universelle ( $\mathrm{kJ} / \mathrm{mol} . \mathrm{K})$.

\section{Analyses statistiques}

Tous les essais de séchage ont été réalisés en triple, soit un total de 18 (6 x 3) séries de séchage. Par la suite, un programme de calcul informatique basé sur la méthode du gradient a été développé et implémenté dans le logiciel MatLab R2016a pour le calcul des coefficients, paramètres des modèles empiriques et des paramètres statistiques de choix de modèles.

Tableau 1 : Domaine expérimental.

\begin{tabular}{rrr}
\hline & \multicolumn{2}{r}{$\begin{array}{r}\text { Valeurs } \\
\text { codées }\end{array} /$ réelles } \\
\hline Paramètres & -1 & +1 \\
& & \\
$\mathbf{X}_{\mathbf{1}}$ & 1 & 1,5 \\
(épaisseur cm) & & \\
$\mathbf{X}_{\mathbf{2}}$ & 40 & \\
\hline (Température $\left.^{\circ} \mathbf{C}\right)$ & 50 & \\
& 60 & \\
\hline
\end{tabular}

Tableau 2 : Nomenclature.

\begin{tabular}{ll}
\hline & Classification \\
\hline $\mathrm{Y}$ & Vitesse normée \\
$\mathrm{a}, \mathrm{b}, \mathrm{c}$ & Coefficient de séchage \\
$\mathrm{EQM}$ & Erreur quadratique moyen \\
$\mathrm{k}, \mathrm{g}, \mathrm{n}, \mathrm{h}$ & Constantes de séchage \\
$\mathrm{N}$ & Nombre d'observation \\
$\mathrm{R}^{2}$ & Coefficient de détermination \\
$\mathrm{X}^{*}$ & Teneur en eau réduite \\
$\mathrm{MS}$ & matière sèche \\
\hline $\mathrm{Symboles}$ Grecs & \\
$\chi^{2}$ & Chi carré réduit \\
\hline Indice & \\
$i$ & Au temps $i$ \\
$e$ & Équilibre \\
0 & Initiale \\
exp & Expérimentale \\
pre & prédictive \\
\hline
\end{tabular}




\section{RESULTATS}

L'évolution de la perte différentielle de la masse des échantillons au cours du séchage est illustrée par la Figure 1. Cette perte de masse présente une allure générale décroissante tout au long du séchage des tranches de mangues. Trois (03) phases de séchage sont observées. La première (01) qualifié de mise en température s'observe pendant les trois (03) premières heures de séchage pour toutes les épaisseurs ( 1 et $1,5 \mathrm{~cm}$ ) et températures $\left(40,50\right.$ et $\left.60{ }^{\circ} \mathrm{C}\right)$. La seconde phase se constate de la $3^{\text {ème }}$ à la $9^{\text {ème }}$ heure de séchage à $\mathrm{T}=60^{\circ} \mathrm{C}$ pour les épaisseurs 1 et 1,5 $\mathrm{cm}$; à la $14^{\text {ème }}$ heure pour les épaisseurs 1 et 1,5 $\mathrm{cm}$ à $\mathrm{T}=50^{\circ} \mathrm{C}$ et jusqu' à la $17^{\mathrm{mme}}$ heure pour les épaisseurs 1 et $1,5 \mathrm{~cm}$ à $\mathrm{T}=40^{\circ} \mathrm{C}$. C'est la phase de séchage à vitesse constante. La phase de ralentissement commence après la fin de la seconde phase et ce jusqu'en fin de séchage. Elle intervient entre : $16 \mathrm{~h}$ et $18 \mathrm{~h}, 23 \mathrm{~h}$ et $27 \mathrm{~h}$ et $29 \mathrm{~h}$ et $33 \mathrm{~h}$ de séchage pour les températures 60 , 50 et $40{ }^{\circ} \mathrm{C}$ et par niveau d'épaisseur. Les vitesses de séchage sont plus importantes pour les températures élevées et pour la petite épaisseur. Les vitesses moyennes de séchage déterminées sont exprimées en $\mathrm{g} \mathrm{H}_{2} \mathrm{O} / \mathrm{g}$.MS/h et sont les suivantes :

- 0,333 et 0,294 respectivement pour les épaisseurs 1 et $1,5 \mathrm{~cm}$ au cours du séchage à $\mathrm{T}=60^{\circ} \mathrm{C}$.

- 0,217 et 0,185 respectivement pour les épaisseurs 1 et $1,5 \mathrm{~cm}$ au cours du séchage à $\mathrm{T}=50^{\circ} \mathrm{C}$.

- 0,172 et 0,152 respectivement pour les épaisseurs 1 et $1,5 \mathrm{~cm}$ au cours du séchage à $\mathrm{T}=40^{\circ} \mathrm{C}$.

Les courbes caractéristiques de séchage (CCS) correspondantes ont été établies en représentant les vitesses de séchage normées $(\mathrm{Y})$ en fonction des teneurs en eau réduites $\left(X^{*}\right)$. Les CCS permettent d'obtenir les équations de vitesses de séchage sous la forme de polynômes de degré 3 . Avec des coefficients de déterminations supérieurs à $0,95(0,982$;
$0,993 ; 0,984 ; 0,985 ; 0,987$ et 0,982$)$ et proches de l'unité (1).

Les expressions mathématiques obtenues sont:

$$
\begin{array}{ll}
- & Y_{40 / 1}=-15,462 X^{* 3}+12,757 X^{* 2}- \\
& 1,1619 X^{*}+0,1822 \\
- & Y_{40 / 1,5}=-25,251 X^{* 3}+19,182 X^{* 2}- \\
& 1,9836 X^{*}+0,1982 \\
- & Y_{50 / 1}=-19,643 X^{* 3}+17,388 X^{* 2}-2,753 X^{*} \\
& +0,1851 \\
- & Y_{50 / 1,5}=-15,978 X^{* 3}+13,875 X^{* 2}- \\
& 1,9005 X^{*}+0,1736 \\
- & Y_{60 / 1}=-8,4012 X^{* 3}+10,32 X^{* 2}-2,5938 X^{*} \\
& +0,2934 \\
- & Y_{60 / 1,5}=-7,4725 X^{* 3}+8,9453 X^{* 2}- \\
& 2,0555 X^{*}+0,2667
\end{array}
$$

Le Tableau 4 présente les coefficients, paramètres des huit (08) modèles de séchage en couche mince ainsi que les paramètres statistiques de précision du lissage des modèles que sont: le coefficient de détermination $\left(\mathrm{R}^{2}\right)$, le Chi carré $\left(\chi^{2}\right)$ réduit, l'erreur quadratique moyenne (EQM). L'analyse du Tableau 4 révèle que les valeurs du paramètre $\mathrm{R}^{2}$ évoluent de 0,9570 à 0,9922 ; 0,8189 à 0,$9424 ; 0,8905$ à 0,$9428 ; 0,9904$ à 0,$9984 ; 0,9843$ à 0,$9965 ; 0,9879$ à 0,9977 , 0,9181 à 0,9963 et 0,8052 à 0,9908 respectivement pour les huit modèles (approche diffusion, deux-termes, Henderson et Pabis Modifié, Logarithmique, MiddiliKucuk, Newton, Page et Verma et al.). Aussi, les valeurs de $\chi^{2}$ réduit fluctuent entre 7,42 e05 et 5,89 e-04 pour le modèle de l'approche de diffusion ; entre 0,0536 et 0,1328 au niveau du modèle deux-terme; de 0,0887 à 0,3108 avec le modèle d'Henderson et Pabis Modifié ; entre $\quad 1,58 \mathrm{e}-07$ et $4,56 \mathrm{e}-06$ pour le modèle Logarithmique. En ce qui concerne le modèle de Middili-Kucuk, les valeurs sont entre 1,238 e-07 et 1,53 e-06. Les modèles Newton, Page et Verma et al., ont permis d'obtenir les valeurs $\mathrm{du} \chi^{2}$ réduit respectivement entre $3,42 \mathrm{e}-07$ et $1,24 \mathrm{e}-05 ; 7,12$ e- 12 et $1,08 \mathrm{e}-07$. Les valeurs des EQM enregistrées sont de: 0,0032 à 0,$0118 ; 0,1139-0,1768 ; 0,1466$ à 0,2704 ; 
$1,95 \mathrm{e}-07$ à 0,$0010 ; 1,71 \mathrm{e}-04$ à 0,$0016 ; 2,33 \mathrm{e}-$ 04 à 0,$0017 ; 1,31 \mathrm{e}-04$ à $1,59 \mathrm{e}-04$ et 0,0051 à 0,0217 respectivement pour ces modèles.

Ces valeurs obtenues sont assez faibles dans l'ensemble. Sur la base de ce que le modèle qui décrit le mieux la cinétique de séchage sur couche mince est celui qui présente la valeur la plus élevée du coefficient de détermination $\left(\mathrm{R}^{2}\right)$, premier critère de choix ; avec de faibles valeurs de $\chi^{2}$ réduit et EQM pour le second critère. Le modèle logarithmique est retenu pour le séchage conductif des tranches de mangue tel qu'illustré sur la Figure 2. L'estimation du coefficient de diffusion et de l'énergie d'activation lors du séchage des tranches de mangues sont consignés dans le Tableau 5. Les valeurs des coefficients de diffusions calculés sont comprises entre 8,615 e-09 et 9,155 e-09 $\mathrm{m}^{2} \mathrm{~s}^{-1}$. L'énergie d'activation a évolué de 22,430 à $25,358 \mathrm{KJ} / \mathrm{mol}$.

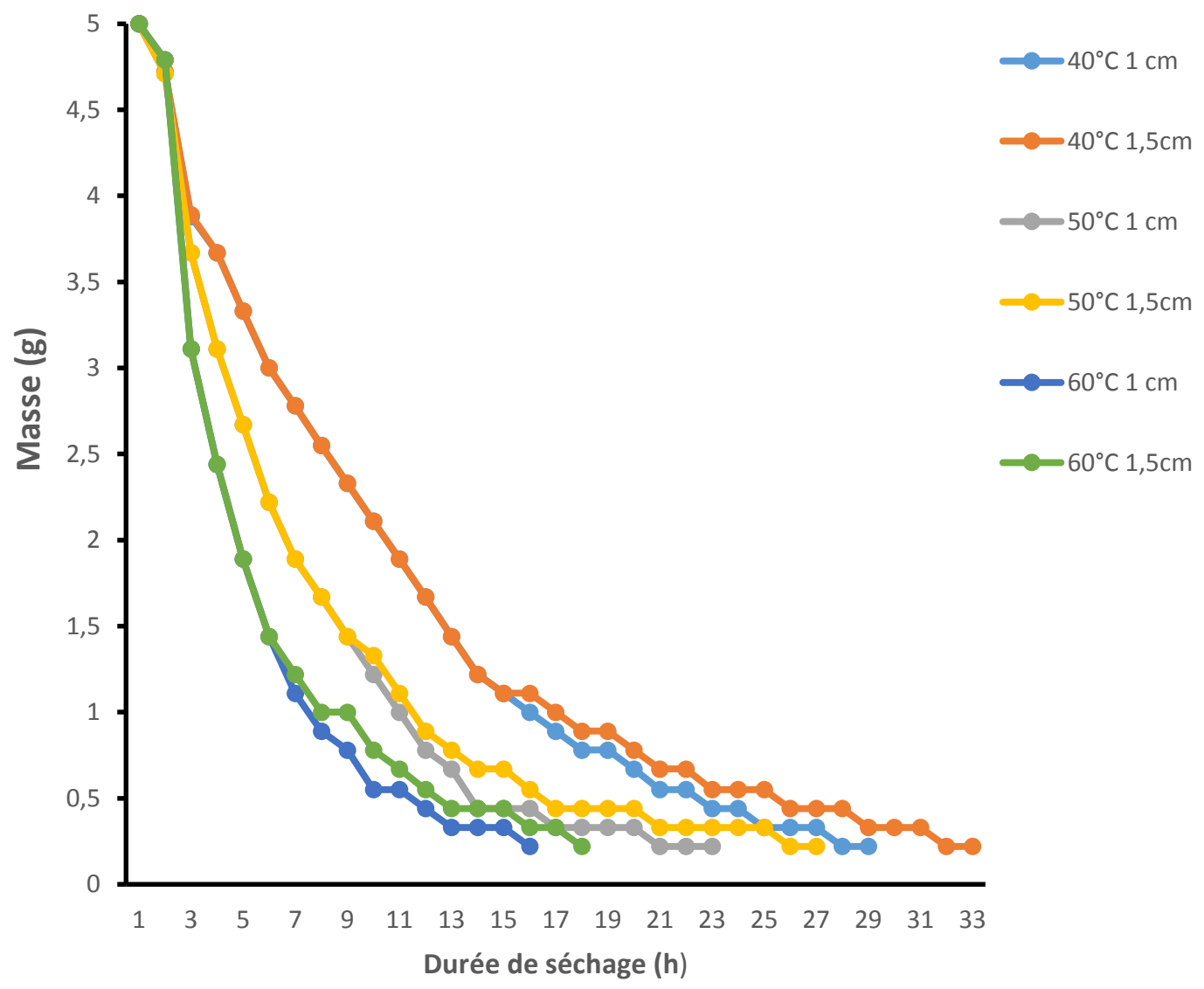

Figure 1 : Evolution de la perte différentielle de masse des tranches de mangue au cours du séchage à différentes températures. 
N. V. ABOUO et al. / Int. J. Biol. Chem. Sci. 14(7): 2476-2490, 2020

Tableau 4 : Valeurs des coefficients, paramètres et critères statistiques issus de la modélisation.

\begin{tabular}{|c|c|c|c|c|c|c|c|c|c|c|c|c|c|}
\hline \multirow{2}{*}{$\begin{array}{l}\text { Modèles } \\
\text { Utilisés }\end{array}$} & \multirow{2}{*}{$\frac{{ }^{\circ} \mathrm{C}}{\mathrm{cm}}$} & \multicolumn{9}{|c|}{ Paramètres et coefficients des modèles de la présente étude } & \multicolumn{3}{|c|}{ Critères statistiques } \\
\hline & & $\mathbf{a} / \mathbf{A} 1$ & b & c & g & $\mathbf{h}$ & $\mathbf{k}$ & k0 & $\mathbf{k 1}$ & $\mathbf{n}$ & $\mathbf{R}^{2}$ & $\chi^{2}$ & EQM \\
\hline & $40 / 1$ & 0,1855 & 0,2981 & - & - & - & 0,1416 & - & - & - & 0,9922 & $5,32 \mathrm{e}-04$ & 0,0036 \\
\hline & $40 / 1,5$ & 0,1857 & 0,298 & - & - & - & 0,1441 & - & - & - & 0,9896 & $4,35 \mathrm{e}-04$ & 0,0032 \\
\hline Approche & $50 / 1$ & 0,1877 & 0,2978 & - & - & - & 0,1419 & - & - & - & 0,9759 & $1,63 \mathrm{e}-04$ & 0,0063 \\
\hline \multirow[t]{5}{*}{ Diffusion } & $50 / 1,5$ & 0,1887 & 0,2973 & - & - & - & 0,1494 & - & - & - & 0,9673 & $7,42 \mathrm{e}-05$ & 0,0042 \\
\hline & $60 / 1$ & 0,1929 & 0,2968 & - & - & - & 0,1469 & - & - & - & 0,9635 & 5,89 e- 04 & 0,0118 \\
\hline & $60 / 1,5$ & 0,194 & 0,2964 & - & - & - & 0,1524 & - & - & - & 0,9570 & $3,63 \mathrm{e}-04$ & 0,0093 \\
\hline & $40 / 1$ & 3,0175 & 4.2668 & - & - & - & - & 0,0011 & 9,38 e-04 & - & 0,9424 & 0,0536 & 0,1139 \\
\hline & $40 / 1,5$ & 3,1407 & 4,4103 & - & - & - & - & 9,59 e-04 & 8,12 e-04 & - & 0,9378 & 0,0544 & 0,1147 \\
\hline Deux- & $50 / 1$ & 2,6915 & 3,9054 & - & - & - & - & 0,0015 & 0,0013 & - & 0,9194 & 0,0737 & 0,1329 \\
\hline \multirow[t]{5}{*}{ Termes } & $50 / 1,5$ & 3,0568 & 4,3442 & - & - & - & - & 0,001 & 8,68 e-04 & - & 0,9174 & 0,0687 & 0,1287 \\
\hline & $60 / 1$ & 2,4941 & 3,6858 & - & - & - & - & 0,0018 & 0,0017 & - & 0,9159 & 0,1328 & 0,1768 \\
\hline & $60 / 1,5$ & 2,7122 & 3,9715 & - & - & - & - & 0,0015 & 0,0013 & - & 0,8189 & 0,1241 & 0,1714 \\
\hline & $40 / 1$ & 7,4492 & 1,8569 & 0,0657 & 0,0021 & 0,0021 & $6,21 \mathrm{e}-04$ & - & - & - & 0,9428 & 0,0892 & 0,1469 \\
\hline & $40 / 1,5$ & 7,5686 & 1,9906 & 0,0707 & 0,0018 & 0,0018 & 5,50 e- 04 & - & - & - & 0,9321 & 0,0887 & 0,1466 \\
\hline Henderson & $50 / 1$ & 7,3486 & 1,6626 & 0,0584 & 0,0026 & 0,0026 & 6,89 e-04 & - & - & - & 0,9196 & 0,1418 & 0,1843 \\
\hline Pabis & $50 / 1,5$ & 7,7188 & 2,053 & 0,0729 & 0,0017 & 0,0017 & $4,72 \mathrm{e}-04$ & - & - & - & 0,8905 & 0,123 & 0,1722 \\
\hline modifié & $60 / 1$ & 7,5699 & 1,7075 & 0,0598 & 0,0024 & 0,0024 & $5,51 \mathrm{e}-04$ & - & - & - & 0,9152 & 0,3108 & 0,2704 \\
\hline
\end{tabular}


N. V. ABOUO et al. / Int. J. Biol. Chem. Sci. 14(7): 2476-2490, 2020

\begin{tabular}{|c|c|c|c|c|c|c|c|c|c|c|c|c|c|}
\hline & $60 / 1,5$ & 7,7724 & 1,9344 & 0,0683 & 0,0019 & 0,0019 & 4,48 e-04 & - & - & - & 0,898 & 0,2715 & 0,2536 \\
\hline \multirow{6}{*}{$\begin{array}{l}\text { Logarith- } \\
\text { mique }\end{array}$} & $40 / 1$ & 0,708 & - & 0,1298 & - & - & - & - & 0,1284 & - & 0,9984 & $2,43 \mathrm{e}-07$ & $2,42 \mathrm{e}-04$ \\
\hline & $40 / 1,5$ & 0,6953 & - & 0,1312 & - & - & - & - & 0,1296 & - & 0,9984 & 1,58 e- 07 & 1,95 e- 07 \\
\hline & $50 / 1$ & 0,6121 & - & 0,1411 & - & - & - & - & 0,137 & - & 0,9981 & $1,25 \mathrm{e}-06$ & $5,47 \mathrm{e}-04$ \\
\hline & $50 / 1,5$ & 0,5597 & - & 0,1476 & - & - & - & - & 0,1414 & - & 0,9987 & $2,02 \mathrm{e}-07$ & $2,20 \mathrm{e}-04$ \\
\hline & $60 / 1$ & 0,4026 & - & 0,1684 & - & - & - & - & 0,1532 & - & 0,9988 & 4,56 e- 06 & 0,001 \\
\hline & $60 / 1,5$ & 0,3541 & - & 0,1753 & - & - & - & - & 0,1563 & - & 0,9985 & 1,37 e-06 & 5,69 e- 04 \\
\hline \multirow{6}{*}{$\begin{array}{l}\text { Middili \& } \\
\text { Kucuk }\end{array}$} & $40 / 1$ & 1,0567 & 0,0143 & - & - & - & 0,1286 & - & - & $\begin{array}{c}0,71 \\
65\end{array}$ & 0,9882 & $1,23 \mathrm{e}-06$ & 5,47 e- 04 \\
\hline & $40 / 1,5$ & 1,0591 & 0,0143 & - & - & - & 0,1286 & - & - & $\begin{array}{c}0,71 \\
75\end{array}$ & 0,9906 & 1,48 e-06 & 5,99 e- 04 \\
\hline & $50 / 1$ & 1,0601 & 0,0177 & - & - & - & 0,1284 & - & - & $\begin{array}{c}0,71 \\
92\end{array}$ & 0,9965 & $1,23 \mathrm{e}-07$ & $1,71 \mathrm{e}-04$ \\
\hline & $50 / 1,5$ & 1,0678 & 0,0181 & - & - & - & 0,1282 & - & - & $\begin{array}{c}0,72 \\
28\end{array}$ & 0,9968 & $1,53 \mathrm{e}-06$ & 6,08 e- 04 \\
\hline & $60 / 1$ & 1,0725 & 0,0244 & - & - & - & 0,1276 & - & - & $\begin{array}{c}0,72 \\
81\end{array}$ & 0,9866 & 1,06 e- 05 & 0,0016 \\
\hline & $60 / 1,5$ & 1,0787 & 0,025 & - & - & - & 0,1274 & - & - & $\begin{array}{c}0,73 \\
13\end{array}$ & 0,9843 & 1,38 e- 06 & 5,72 e- 04 \\
\hline \multirow{6}{*}{ Newton } & $40 / 1$ & - & - & - & - & - & 0,1299 & - & - & - & 0,9879 & $3,42 \mathrm{e}-07$ & $2,87 \mathrm{e}-04$ \\
\hline & $40 / 1,5$ & - & - & - & - & - & 0,1313 & - & - & - & 0,9897 & $2,25 \mathrm{e}-07$ & $2,33 \mathrm{e}-04$ \\
\hline & $50 / 1$ & - & - & - & - & - & 0,1411 & - & - & - & 0,997 & $2,07 \mathrm{e}-06$ & 7,05 e-04 \\
\hline & $50 / 1,5$ & - & - & - & - & - & 0,1474 & - & - & - & 0,9977 & 3,44 e- 07 & $2,88 \mathrm{e}-04$ \\
\hline & $60 / 1$ & - & - & - & - & - & 0,1672 & - & - & - & 0,9936 & 1,24 e- 05 & 0,0017 \\
\hline & \multicolumn{13}{|c|}{2484} \\
\hline
\end{tabular}


N. V. ABOUO et al. / Int. J. Biol. Chem. Sci. 14(7): 2476-2490, 2020

\begin{tabular}{|c|c|c|c|c|c|c|c|c|c|c|c|c|c|}
\hline & $60 / 1,5$ & - & - & - & - & - & 0,1734 & - & - & - & 0,994 & $4,01 \mathrm{e}-06$ & 9,75 e- 04 \\
\hline \multirow{6}{*}{ Page } & $40 / 1$ & - & - & - & - & - & - & - & 0,1751 & $\begin{array}{c}1,13 \\
19\end{array}$ & 0,9181 & $4,14 \mathrm{e}-11$ & 3,16 e-06 \\
\hline & $40 / 1,5$ & - & - & - & - & - & - & - & 0,1744 & $\begin{array}{c}1,13 \\
49\end{array}$ & 0,9235 & $1,84 \mathrm{e}-11$ & $2,11 \mathrm{e}-06$ \\
\hline & $50 / 1$ & - & - & - & - & - & - & - & 0,1518 & $\begin{array}{c}1,22 \\
86\end{array}$ & 0,969 & 5,18 e- 10 & $1,11 \mathrm{e}-05$ \\
\hline & $50 / 1,5$ & - & - & - & - & - & - & - & 0,1443 & $\begin{array}{c}1,25 \\
71\end{array}$ & 0,9694 & 7,12 e- 12 & $1,31 \mathrm{e}-06$ \\
\hline & $60 / 1$ & - & - & - & - & - & - & - & 0,0466 & $\begin{array}{c}1,66 \\
5\end{array}$ & 0,9963 & $1,08 \mathrm{e}-07$ & 1,59 e- 04 \\
\hline & $60 / 1,5$ & - & - & - & - & - & - & - & 0,0331 & $\begin{array}{c}1,73 \\
65\end{array}$ & 0,9863 & 4,89 e- 08 & 1,07 e- 04 \\
\hline \multirow{6}{*}{ Verma et al., } & $40 / 1$ & 0,7212 & - & - & 0,2659 & - & 0,0265 & - & - & - & 0,9908 & $1,08 \mathrm{e}-04$ & 0,0051 \\
\hline & $40 / 1,5$ & 0,7422 & - & - & 0,2658 & - & 0,0258 & - & - & - & 0,9886 & $1,06 \mathrm{e}-04$ & 0,0051 \\
\hline & $50 / 1$ & 7080 & - & - & 0,2958 & - & 0,0193 & - & - & - & 0,9873 & 3,59 e- 04 & 0,0093 \\
\hline & $50 / 1,5$ & 0,7579 & - & - & 0,2994 & - & 0,017 & - & - & - & 0,9763 & $2,92 \mathrm{e}-04$ & 0,0084 \\
\hline & $60 / 1$ & 0,6225 & - & - & 0,3898 & - & $-0,0098$ & - & - & - & 0,8635 & 0,002 & 0,0217 \\
\hline & $60 / 1,5$ & 0,6265 & - & - & 0,3946 & - & $-0,0121$ & - & - & - & 0,8052 & 0,0018 & 0,0207 \\
\hline
\end{tabular}




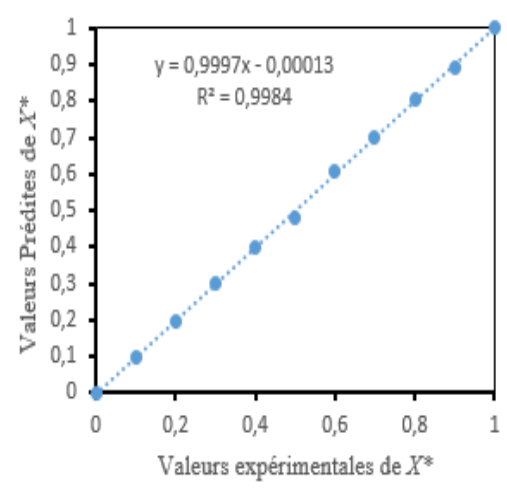

a) $\mathrm{T}=40^{\circ} \mathrm{C}$ et $\mathrm{E}=1 \mathrm{~cm}$

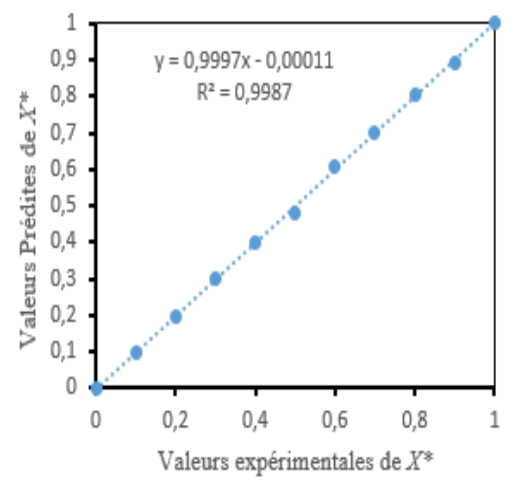

d) $\mathrm{T}=50^{\circ} \mathrm{C}$ et $\mathrm{E}=1,5 \mathrm{~cm}$

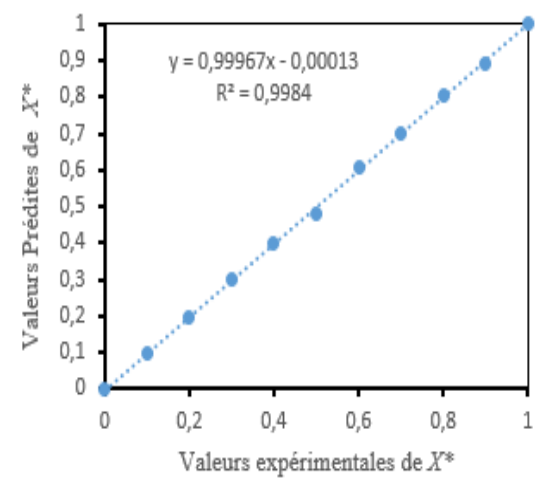

b) $\mathrm{T}=40^{\circ} \mathrm{C}$ et $\mathrm{E}=1,5 \mathrm{~cm}$

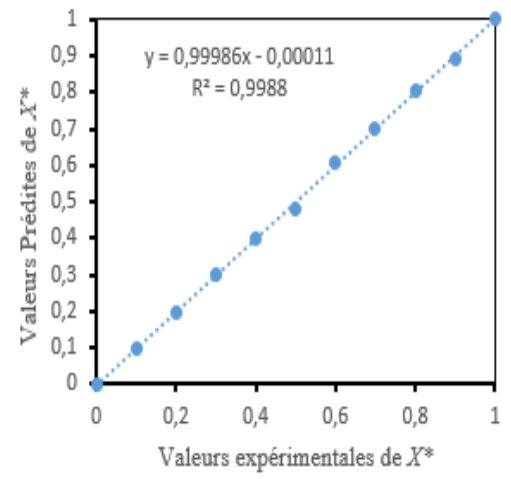

e) $\mathrm{T}=60^{\circ} \mathrm{C}$ et $\mathrm{E}=1 \mathrm{~cm}$

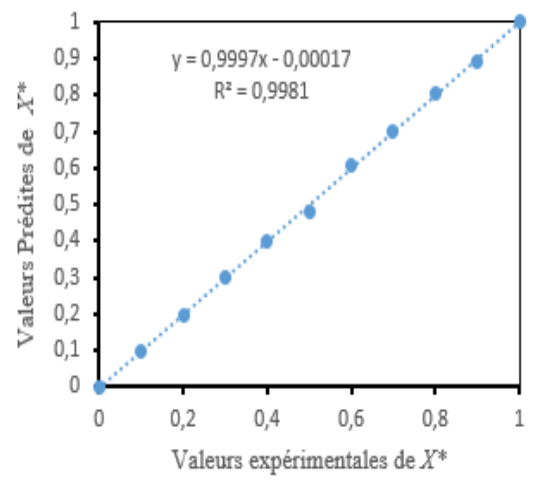

c) $\mathrm{T}=50^{\circ} \mathrm{C}$ et $\mathrm{E}=1 \mathrm{~cm}$

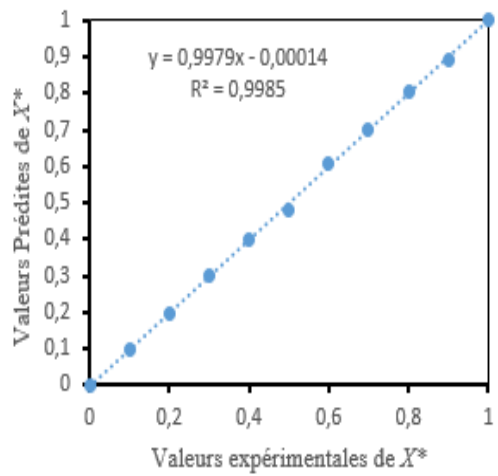

f) $\mathrm{T}=60^{\circ} \mathrm{C}$ et $\mathrm{E}=1,5 \mathrm{~cm}$

Figure 2 : Représentation graphique du $\mathrm{R}^{2}$ du modèle Logarithmique pour les températures (40, 50, $60^{\circ} \mathrm{C}$ ) et épaisseurs (1 et $\left.1,5 \mathrm{~cm}\right)$.

Tableau 5 : Estimation de coefficient de diffusion (Deff) et de l'énergie d'activation (Ea) des tranches de mangues séchées.

\begin{tabular}{cccc}
\hline $\begin{array}{c}\text { Température } \\
\left({ }^{\mathbf{C}} \mathbf{C}\right)\end{array}$ & Epaisseur $(\mathbf{c m})$ & $\begin{array}{c}\text { Deff } \\
\left(\mathbf{m}^{2} \mathbf{s}^{-\mathbf{1}}\right)\end{array}$ & $\begin{array}{c}\text { Ea } \\
\left(\mathbf{K J m o l}^{-\mathbf{1}}\right)\end{array}$ \\
\hline 40 & 1 & $8,687 \mathrm{e}-09$ & 22,430 \\
40 & 1,5 & $8,615 \mathrm{e}-09$ & 22,618 \\
\hline 50 & 1 & $9,026 \mathrm{e}-09$ & 23,931 \\
50 & 1,5 & $8,907 \mathrm{e}-09$ & 24,251 \\
\hline 60 & 1 & $9,155 \mathrm{e}-09$ & 25,325 \\
60 & 1,5 & $9,143 \mathrm{e}-09$ & 25,358 \\
\hline
\end{tabular}




\section{DISCUSSION}

La courbe de séchage des tranches de mangue présente une allure générale décroissante avec trois (03) phases qui se dégagent. La phase de mise en température, la première s'observe pendant les trois (03) premières heures de séchage. La seconde se

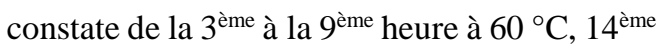
heure à $50^{\circ} \mathrm{C}$ et $17^{\text {ème }}$ heure à $40^{\circ} \mathrm{C}$. La vitesse de séchage des tranches est fonction du couple temps/température comme l'ont indiqué les travaux de Touati (2008), Chekroune (2009), Abouo et al. (2015b) respectivement lors du séchage des feuilles de menthes, de dattes et fèves de cacao. Les vitesses de séchage sont plus importantes pour les températures élevées et pour la faible épaisseur dans tous les cas de figures.

Les courbes caractéristiques de séchage (CCS) correspondantes établies sont des polynômes de degré 3 avec des coefficients de détermination multiple élevés $(0,982 ; 0,993$; 0,$984 ; 0,985 ; 0,987$ et 0,982 ).

Des résultats similaires sont rapportés dans la littérature sur le séchage des menthes et du cacao (Touati, 2008 ; Abouo et al., 2015b). Les paramètres et coefficients de séchage des huit modèles retenus ont permis le lissage des données expérimentales aux données prédites. L'analyse des valeurs du paramètre $\mathrm{R}^{2}$ indique qu'il a évolué de 0,9570 à 0,$9922 ; 0,8189$ 0,$9424 ; \quad 0,8905-0,9428 ; \quad 0,9904-0,9984$; 0,9843-0,9965; 0,9879-0,9977, 0,9181-0,9963 et 0,8052-0,9908 respectivement pour les huit modèles (approche diffusion, deux-termes, Henderson et Pabis Modifié, Logarithmique, Middili-Kucuk, Newton, Page et Verma et al.,). L'analyse des valeurs du paramètre $\mathrm{R}^{2}$ a révélé des valeurs supérieures à 0,80 . Cela traduit ainsi un assez bon ajustement des distributions expérimentales et prédites par les modèles testés dans la présente étude (Karidioula et al., 2018 ; Koko et al., 2018). Aussi, les valeurs de $\chi^{2}$ réduit fluctuent entre 7,42 e-05 et 5,89 e-04 pour le modèle de l'approche de diffusion ; entre 0,0536 et 0,1328 au niveau du modèle
Deux-terme; entre 0,0887 et 0,3108 avec le modèle d'Henderson et Pabis Modifié ; entre 1,58 e-07 et 4,56 e-06 pour le modèle Logarithmique. En ce qui concerne le modèle de Middili-Kucuk, elles sont entre 1,238 e-07 et 1,53 e-06. Les modèles Newton, Page et Verma et al., ont permis d'obtenir les valeurs $\mathrm{du} \chi^{2}$ réduit respectivement de 3,42 e-07 à 1,24 e-05; 7,12 e-12 à 1,08 e-07. Les valeurs des EQM enregistrées sont de : 0,0032 à 0,0118 ; 0,1139 à 0,$1768 ; 0,1466$ à 0,$2704 ; 1,95$ e-07 à 0,$0010 ; 1,71$ e-04 à 0,$0016 ; 2,33$ e-04 à 0,$0017 ; 1,31$ e-04 à 1,59 e-04 et 0,0051 à 0,0217 . Ces valeurs obtenues sont assez faibles dans l'ensemble. Sur la base de ce que le modèle qui décrit le mieux la cinétique de séchage sur couche mince est celui qui présente la valeur la plus élevée du coefficient de détermination $\left(\mathrm{R}^{2}\right)$, premier critère de choix; avec de faibles valeurs de $\chi^{2}$ réduit et EQM, le modèle logarithmique a été retenu pour le séchage conductif des tranches de mangue. Ce modèle a été retenu pour le séchage solaire des fèves de cacao (Akmel et al., 2009). Les valeurs des coefficients de diffusion calculés sont de l'ordre de $10^{-9}$, valeur généralement rencontrée dans le cadre des produits végétaux (Hii et al., 2006; Akmel et al., 2009). Les valeurs obtenues sont proches de ceux rapportés par Mamouda et al. (2019), lors du séchage de la mangue dans un séchoir type solaire par convection forcée. La valeur moyenne du coefficient de diffusion rapportée est de 8,866 e- $09 \mathrm{~m}^{2} / \mathrm{s}$.

\section{Conclusion}

Au terme de cette étude, il ressort que le séchage des tranches de mangues dure entre $16 \mathrm{~h}$ et $18 \mathrm{~h}, 23 \mathrm{~h}$ et $27 \mathrm{~h}$ et enfin entre $29 \mathrm{~h}$ et $33 \mathrm{~h}$ selon les températures $\left(60,50\right.$ et $\left.40{ }^{\circ} \mathrm{C}\right)$ et épaisseurs $(1,5 ; 1 \mathrm{~cm})$ respectives. Les vitesses de séchage sont plus importantes pour les températures élevées et pour la petite épaisseur. Le modèle logarithmique a présenté la valeur du $\mathrm{R}^{2}$ (0,9984; 0,9984; 0,9981; 0,9987; $0,9988 ; 0,9985)$ les plus élevées et les plus 
faibles valeurs de $\chi^{2}$ (2,43 e-07; 1,58 e-07; 1,25 e-06 ; 2,02 e-07; 4,56 e-06 et 1,37 e-06) et EQM $(2,42 \mathrm{e}-04 ; 1,95 \mathrm{e}-07 ; 5,47 \mathrm{e}-04 ; 2,20$ e-04; 0,0010 et 5,69 e-04) pour les températures et épaisseurs correspondantes. Il décrit donc le mieux le séchage conductif des tranches de mangues. Le coefficient de diffusion $\left(8,615\right.$ e-09 à 9,155 e-09 $\left.\mathrm{m}^{2} / \mathrm{s}\right)$ dépend fortement de la température de séchage. L'énergie d'activation moyenne est estimée entre 22,430 et $25,358 \mathrm{KJ} / \mathrm{mol}$.

\section{CONFLIT D'INTERETS}

Les auteurs attestent qu'il n'y a aucun conflit d'intérêts.

\section{CONTRIBUTIONS DES AUTEURS}

NVA est le principal investigateur de l'étude et a participé à toutes les phases du travail. AF et YDN ont contribué à la réalisation de l'expérimentation et à la correction du manuscrit. NEA a assuré la supervision générale des travaux et l'interprétation approfondie des résultats.

\section{REFERENCES}

Abouo NV, Kassi AB, Soro D, Assidjo NE, Amani NG, Yao KB. 2015a. Impact de sechage solaire et microonde sur la qualité du beurre de cacao (Theobroma L.,). Eur. Sci. J., 11(12): 263-273. DOI : http://eujournal.org/index.php/esj/article/ view/5480

Abouo NV, Akmel DC, Kakou KE, Assidjo NE, Amani NG, Yao KB. 2015b. Modelling of thin layer drying kinetics of cocoa bean in microwave oven and sun. Food Environ. Saf., XIV(2) :127-137. DOI : http://fiaold.usv.ro/fiajournal/index.php/FENS/art icle/view/24

Afoakwa E, Kongor J, Takrama J, Budu A. 2013. Changes and nib acidification and biochemical composition during fermentation of pulp pre-conditioned cocoa (Theobroma cocoa) beans. Int.
Food Res. J., 20(4): 1843-1853. DOI: https://www.researchgate.net/publication 1270272202

Afoakwa E. 2008. Cocoa and chocolate consumption- Are there aphrodisiac and other benefits for human health? Afri. $J$. Clinic Nutri., 21(3): 107-113. DOI: $10.1080 / 16070658.2008 .11734163$

Akmel D, Assidjo N, Yao B. 2008. Effet des dispositifs de séchage à l'air libre sur la qualité des fèves de cacao bord champ, Rev. Ivoir. Sci. Technol., 11 : 45-58. DOI : https://revist.net/REVIST_11/REVIST_1 1_3.pdf

Akmel DC, Assidjo NE, Kouame P, Yao KB. 2009. Mathematical Modelling of Sun Drying Kinetics of Thin Layer Cocoa (Theobroma Cacao) Beans. J. Appli. Sci. Res., 5(9): 1110-1116. DOI : https://www.researchgate.net/publication 1228508629

AOAC. 2005. Official Methods of Analysis (18th edn). Association of Official Analytical Chemists: Washington, DC, Moisture Content in Plants, 1: 949.

Assovie KO. 2012. Revue nationale pour identifier les initiatives de valorisation non alimentaire de la mangue en Côte d'ivoire. $\quad N^{\circ} 12 /$ Coleacp Paepard-01/ BNA-12, 43 p.

Belahmidi M, Belghit A, Mrani A, MIR A, Kaoua M. 1993. Approche expérimentale de la cinétique de séchage des produits agro-alimentaires. Rev. Gen. Therm., 380-381: 444-453. DOI : https://www.yumpu.com/fr/document/vi ew/50138514

CBI-Ministry OF Foreign Affairs. 2019. Analyse de la chaine de valeur des fruits transformés au Burkina Faso, Ghana et en Côte d'Ivoire, $178 \mathrm{p}$.

Chekroune M. 2009. Etude comparative de deux types de séchage (convection et microonde) par application des plans d'expériences: Cas des fruits de datte. 
Mémoire de Master, Université M'Hamed Bougara Boumerdes, Algérie, $127 \mathrm{p}$.

Dembele DD, Amari Ler-N'ogn D, Camara B, Grechi I, Rey J-Y, Kone D. 2019. Pre and postharvest assessment of mango anthracnose incidence and severity in the north of Côte d'Ivoire. Int. J. Biol. Chem. Sci., 13(6): 2726-2738. DOI: https://dx.doi.org/10.4314/ijbcs.v13i6.24

Floury J, Le bail A, Pham QT. 2008. A threedimensional numerical simulation of the osmotic dehydration of mango and effect of freezing on the mass transfer rates. J. Food Eng., 85 : 1-11. DOI: 10.1016/j.jfoodeng.2007.06.011

Gowen A, Abu-ghannam N, Oliviera J. 2008. Modelling dehydratation and rehydratation of cooked soybeans subjected to combined microwave-hot air drying. Innov. Food Sci. Emerg., 9: 129137. DOI: 10.1016/j.ifset.2007.06.009

Griva I, Nash G, Sofer A. 2009. Linear and Nonlinear Optimization. Edition SIAM.

Hii CL, Law CL, Cloke M. 2006. Modeling using a new thin layer drying model and product quality of cocoa. JESTEC., 3(1): 192-198.

DOI:

10.1016/j.jfoodeng.2008.06.022

Jurendic T. 2012. Determination of controlling resistance to moisture transfer during drying. J. Food Sci. Technol., 4(1): 34-45. DOI : https://hrcak.srce.hr/84718

Kakou KE, Akmel DC, Abouo NV, Assidjo NE, Niamke LS. 2015. Isotherme d'adsorption d'eau des fèves de cacao (Theobroma cacao L.) marchand. Eur. Sci. J., 11(12): 355-370. DOI: http://eujournal.org/index.php/esj/article/ view/5489

Karidioula D, Akmel DC, Assidjo NE, Trokourey A. 2018. Modélisation du séchage solaire de fèves de cacao par le réseau de neurones artificiel. Int. J. Biol.
Chem. Sci., 12(1): 195-202.

DOI: $10.4314 /$ ijbcs.v12i1.15

Koko AC, Diomande M, Kouame KB, Assidjo NE. 2018. Détermination expérimentale et modélisation des isothermes d'adsorption d'eau des amandes d'Irvingia gabonensis de la région du Haut-Sassandra (Côte d'Ivoire). IOSRJESTFT., 12(2): 50-66. DOI: 10.9790/2402-1202025066

Kone K. 2011. Amélioration de la qualité de la tomate séchée par microondes assisté par air chaud avec pilotage de la puissance spécifique. Thèse de Doctorat, Paris Tech, France, p.1.

Kouadio A, Aw S, Assidjo N, Kouamé P. 2015. Etude de la qualité physico-chimique et mycologique du cacao (Theobroma cacao L.) produit dans les zones de Yamoussoukro et Soubré (Côte d'Ivoire). Int. J. Biol. Chem. Sci., 13(1): 330-340.

Krysiak W. 2011. Effects of convective and microwave roasting on the physicochemical properties of cocoa beans and cocoa butter extracted from this material. Grasas Y. Aceites, 62(4): 467478. DOI: $10.3989 /$ gya. 114910

Mamouda MA, Saidou M, Makinta B. 2019. Drying kinetics of tomato, Okra, Potato and Mango in a forced convective solar tunel dryer. Int. J. Green. Energy, 8(2): 34-44.

DOI: 10.11648/j.ijrse.20190802.12

Midilli A, Kucuk H. 2003. Mathematical modelling of thin layer drying of pistachio by using solar energy. Energy Convers. Manag., 44(7): 11111122. DOI: $10.1016 / \mathrm{S} 0196-$ 8904(02)00099-7

N'goran ZE, Aw S, Assidjo NE, Kouame LP. 2012. Influence des paramètres de l'osmotique déshydratation sur la perte d'eau dans la papaye et la mangue. $J$. Appl. Biosci., 59: 4330-4339. DOI : http://www.m.elewa.org/JABS/2012/59/ 6.pdf 
Nogbou ALI, Akmel DC, Brou KD, Assidjo NE. 2015. Modélisation de la cinétique de séchage des fèves de cacao par des modèles semi-empiriques et par un réseau de neurones artificiels récurrent: cas du séchage microonde par intermittence. Eur. Sci. J., 11(9): 118-133. DOI : https://eujournal.org/index.php/esj/article /view/5283

Nouar M, Decreux Y, Marty O. 2013. Evaluation du potentiel à l'exportation des fruits tropicaux Côte d'ivoire. Programme d'Appui au Commerce et à !'Intégration Régionale (PACIR), $170 \mathrm{p}$.

Roberts J, Kidd D, Padilla-Zakour O. 2008. Drying kinetics of grape seeds, $J$. Food Eng., $\quad$ 89: $\quad 460-465 . \quad$ DOI: 10.1016/j.jfoodeng.2008.05.030

Sorour H, EL-Mesery H. 2014. Effect of microwave and infrared radiation on drying of onion slices. IJRANSS., 2(5): 119-130. DOI : http://oaji.net/articles/2014/4911404713441.pdf

Togrul I, Pehlivan D. 2004. Modeling of thin layer drying of some fruits under open-air sun drying process. $J$.
Food Eng.,

65:

413-425.

DOI: 10.1016/j.jfoodeng.2004.02.001

Touati B. 2008. Etude théorique et expérimentale du séchage solaire des feuilles de la menthe verte (Mentha viridis). Thèse de l'Insttitut National des Sciences Appliquées de Lyon, France, p. 73.

Verma L, Bucklin R, Endan J, Wratten F. 1985. Effects of drying air parameters on rice drying models. Transactions of the ASAE, 28: 296-301. DOI : https://doi.org/10.13031/2013.32245

Yah NM. 2015. Anthracnose de la mangue (Mangifera indica L.) en Côte d'Ivoire: Caractérisation de l'agent pathogène et efficacité in vitro et in vivo de deux. fongicides chimiques. Thèse de Doctorat Unique, Université Nanguy Abrogoua, Côte d'Ivoire, p. 3.

Yaldiz O, Ertekin C, Uzun HB. 2001. Mathematical modelling of thin layer solar drying of sultana grapes. Energy, 26: 457-465. DOI: $10.1016 / \mathrm{S} 0360-$ 5442(01)00018-4. 